\title{
EFFECT OF ATTRACTOR ON THE DESYNCHRONIZATION EVENTS IN COUPLED CHAOTIC CIRCUITS
}

\author{
Gilson F. de O. Junior*, Hugo L. D. De S. Cavalcante ${ }^{\dagger}$, Orlando di Lorenzo*, Martine \\ Chevrollier*, Thierry Passerat de Silans*, Marcos Orí́* \\ *Laboratório de Dinâmica Não-Linear, Caos e Sistemas Complexos, Departamento de Física, \\ Universidade Federal da Paraiba, 58059-900, João Pessoa, PB, Brazil.
}

${ }^{\dagger}$ Departamento de Informática, Universidade Federal da Paraíba, 58059-900, João Pessoa, PB, Brazil.

Emails: gilson@otica.ufpb.br, hugo@otica.ufpb.br, dilorenzo@otica.ufpb.br, martine@otica.ufpb.br, thierry@otica.ufpb.br, oria@otica.ufpb.br

\begin{abstract}
The phenomenon of attractor bubbling consists in incomplete or imperfect synchronization between two coupled chaotic oscillators, which synchronization regime is broken for brief escapes of different sizes. Here, we use as oscillators two chaotic electronic circuits coupled through unidirectional linear feedback in the regime of moderate coupling to study the occurrence of attractor bubbling in this system. For two different dynamical states, the system exhibits different chaotic attractors. Analysing temporal series, we build empirical distributions of the amplitudes of desynchronization events for different values of the coupling parameter. We observe that in the regime of attractor bubbling the distributions are characterized by a heavy tail, bearing similarity to the ones observed in complex systems with self-organized criticality. A given heavy-tailed distribution is exhibited by the two chaotic states for different coupling strengths. We explain this effect of the attractor shape on the statistics of the desynchronization as caused by a region of instability, which is more often visited for one of the attractors than it is by the other.
\end{abstract}

Keywords- Chaotic dynamic, electronic circuits, heavy-tail statistics, chaotic attractor, attractor shape, attractor bubbling

\section{Introduction}

Because trajectories of chaotic systems are exponentially sensitive to perturbations in phase-space (Boccaletti et al., 2002), the study of chaotic synchronization (Pecora and Carroll, 1990) is more interesting than that of periodic states (Acebrón et al., 2005). Indeed, one can find several generalizations to the concept of chaotic synchronization (Rulkov et al., 1995; Rosenblum et al., 1996; Kocarev and Parlitz, 1996). In special, mismatched parameters or noise can make a system exhibit imperfect synchronization. In such a situation the systems are most of the time synchronized and eventually bursts of desynchronization occur, the so-called attractor bubbling (Heagy et al., 1995; Gauthier and Bienfang, 1996; Venkataramani et al., 1996). Here we study a nonlinear dynamical system comprised of two electronic oscillators governed by second-order differential equations and driven by an external periodic force (Gonçalves and Neto, 2011; Junior et al., 2013). Besides high-quality sinchronization for strong coupling regime and uncorrelation for weak coupling regime, for an intermediate range of values of the coupling level the system presents imperfect chaotic synchronization, exhibiting, then, attractor bubbling, where the difference between the state variables observed in the coupled elements shows long intervals of low values interspersed with sudden and brief departures to large values, which we call bubbles, bursts, or desynchronization events. The distribution of burst sizes follows a heavy-tailed histogram, with large events becoming increasingly rare, similar to power-law (Pareto) distributions found in Lévy Flights. We related heavy-tailed distributions appearing in complex systems to the statistics of desynchronization events in simple coupled chaotic systems (Ashwin et al., 1994; Cavalcante et al., 2013). We observe that the system studied here may exhibit two different chaotic states when the values of the system parameters are changed. We compare the occurrence of attractor bubbling for different "shapes" of the attractor, corresponding to two distinct chaotic states, and we show that in one of these states the occurrence of bursts of desynchronization is more probable.

\section{Circuit description}

The schematic diagram of one of the chaotic oscillators (the drive system) is shown in Fig. 1. It is comprised of resistors, capacitors, diodes and operational amplifiers. The values of the components used in the circuit are: $R_{1}=46.5 \mathrm{k} \Omega, \mathrm{R}$ $=14.86 \mathrm{k} \Omega, R_{2}=14.85 \mathrm{k} \Omega, R_{E}=14.86 \mathrm{k} \Omega, R_{L}$ $=512 \Omega, R_{3}=14.85 \mathrm{k} \Omega, C_{1}=14.73 \mathrm{nF}, C_{2}=$ $14.83 \mathrm{nF}, C_{3}=14.83 \mathrm{nF}$. The diodes $D_{1}$ and $D_{2}$ are model $2 \mathrm{~N} 4148$ and the operational amplifiers OP1 and OP2 are LF411CN.

Analysis of Kirchhoff's laws to our circuit reveals that the dynamical state of the circuit can be expressed in terms of the voltages $V_{1 d}, V_{2 d}$ and $V_{E}$, which obey a second-order differential equation with an external pumping (Gonçalves and Neto, 2011). This second-order differential equa- 


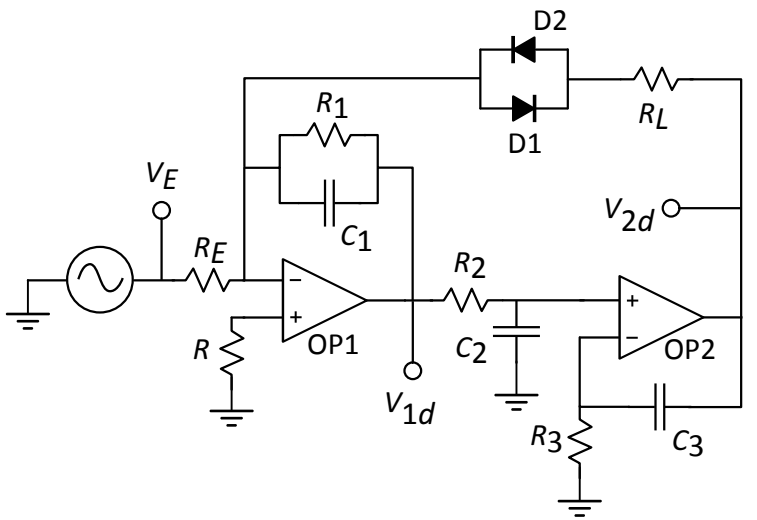

Figure 1: Schematic diagram of the electronic circuit for one chaotic oscillator. This circuit implements a second-order differential equation driven by external sinusoid signal.

tion can be written as two first-order equations:

$$
\begin{aligned}
\dot{V}_{1 d} & =-\alpha V_{1 d}-\beta I\left(V_{2 d}\right)-\theta V_{E} \\
\dot{V}_{2 d} & =\sigma V_{1 d}
\end{aligned}
$$

where $V_{1 d}$ and $V_{2 d}$ are the voltages at the outputs of OP1 and OP2, respectively, $V_{E}=A \sin \omega t, \omega=$ $2 \pi f, \alpha=1 /\left(R_{1} C_{1}\right), \beta=1 /\left(C_{1}\right), \theta=1 /\left(R_{E} C_{1}\right)$, $\sigma=1 /\left(R_{2} C_{2}\right)$ and $I\left(V_{2 d}\right)$ is the current through the diodes D1 and D2 and going out of the inverting input of OP1. It is approximately given by

$$
I(V)=\left\{\begin{array}{cc}
(V+0.7) / \mathrm{R}_{\mathrm{L}}, & \text { if } V_{2 d}<-0.7 \\
0, & \text { if }\left|V_{2 d}\right| \leq 0.7 \\
(V-0.7) / \mathrm{R}_{\mathrm{L}}, & \text { if } V_{2 d}>0.7
\end{array}\right.
$$

and this function is depicted in Fig. 2 .

Let us recall that, for a continuous, autonomous system to be chaotic its dynamics needs i) to be embedded in at least a three-dimensional (3D) phase-space (Strogatz, 1994), and ii) to have a nonlinear term. The nonlinearity of our system is in the current due to the diodes, D1 and D2, and it is given by Eq. (3). The phase of the external forcing can be recast as a dynamical variable that provides for the third dimension.

Changing the amplitude and the frequency of the external signal we control the dynamical behavior of the system, turning it periodic or chaotic. Fig. 3 shows two projections in the plane $V_{1 d^{-}} V_{2 d}$ of a chaotic trajectory in the $3 \mathrm{D}$ attractor of the drive system for different values parameters: external pumping of (a) $A=4.0 \mathrm{~V}$ and $f=$ $770 \mathrm{~Hz}$ (attractor A) and (b) $A=4.0 \mathrm{~V}$ and $f=$ $980 \mathrm{~Hz}$ (attractor B). The trajectories of the attractor in Fig. 3(a) pass close to a central region $\left(V_{1 d}=V_{2 d}=0 \mathrm{~V}\right)$ of phase space. Whereas the trajectories of attractor in Fig. 3(b) do not visit that region of phase space. As we show later, synchronization is harder for the attractor shown in Fig. 3(a), thus suggesting that the central region

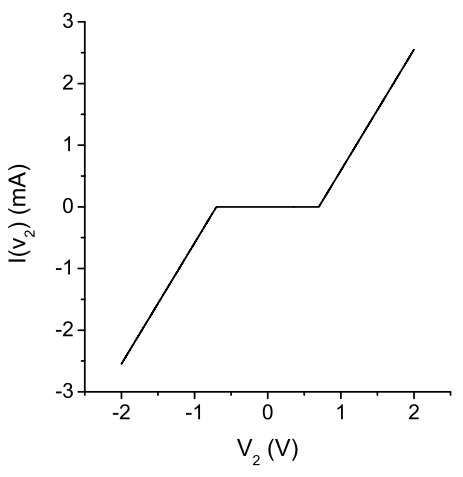

Figure 2: Nonlinear current through a pair of antiparallel diodes. This is the piecewise-linear current given by Eq. (3).

around $V_{1 d}=V_{2 d}=0 \mathrm{~V}$ is unstable (Gauthier and Bienfang, 1996).
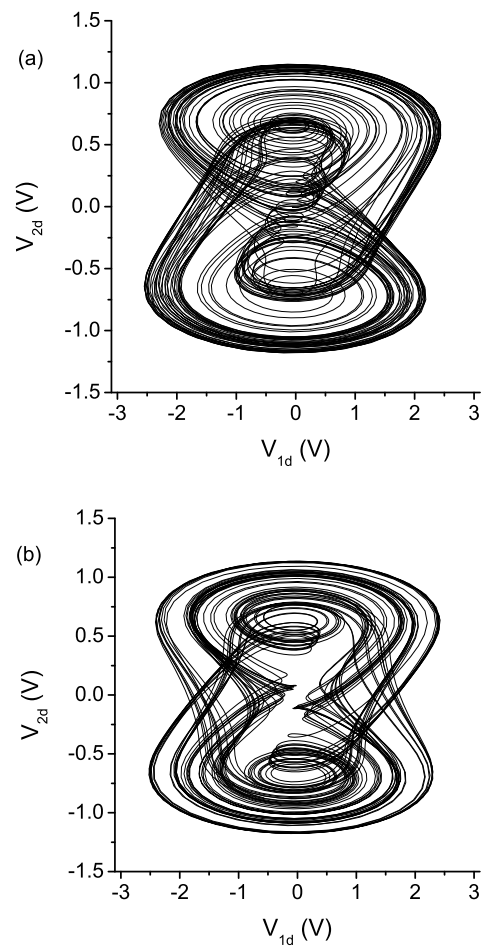

Figure 3: Chaotic trajectorys in the plane $V_{1 d^{-}} V_{2 d}$, obtained with external pumping of (a) $A=4.0 \mathrm{~V}$ and $f=770 \mathrm{~Hz}$ (attractor A), (b) $A=4.0 \mathrm{~V}$ and $f=980 \mathrm{~Hz}$ (attractor B). The values of the other parameters are given in the text.

In Fig. 4 we show the response circuit together with the coupling circuit, where the feedback signal is produced by a subtractor (operationalamplifier OP3) whose input voltages are $V_{1 d}$ and $V_{1 r}$ and whose output voltage is $V_{1 d}-V_{1 r}$. This feedback signal is added to the dynamics of the response subsystem providing the coupling between the response and the drive. The parameter that measures the coupling level between the circuits 
is $\epsilon=R_{2} / R_{R E}$, which is tunable by resistor $R_{R E}$. We set the parameters such that the circuits were both in the same chaotic state when uncoupled $(\epsilon=0)$.

The components of the response and subtractor circuits have the following values: $R_{1}, R, R_{2}$, $R_{3}, R_{E}, C_{1}$, the diodes $D_{1}=D_{2}$ and the operational amplifiers OP1, OP2 and OP3 are equal to the ones used in the drive circuit. $R_{L}=511$ $\Omega, R_{4}=R_{5}=R_{6}=R_{7}=14.85 \mathrm{k} \Omega, C_{2}=14.87$ $\mathrm{nF}, C_{3}=14.87 \mathrm{nF}$. The same sinusoidal signal from a function generator, with amplitude of 4.0 $\mathrm{V}$ and frequency $770 \mathrm{~Hz}$ (attractor A) or $980 \mathrm{~Hz}$ (attractor B) was applied to both the drive and response systems. Notice that the third dimension does not contribute to the distance, as both oscillators share the same value of $V_{E}$ (the complete system is indeed $5 \mathrm{D}$, instead of $6 \mathrm{D}$ ).

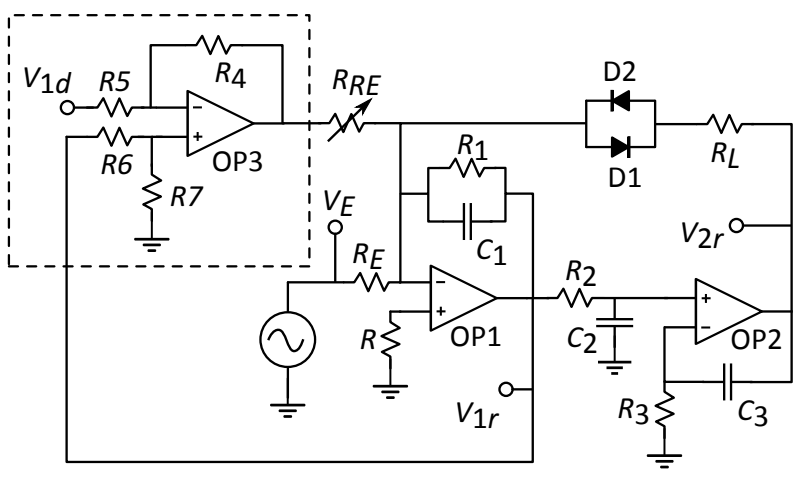

Figure 4: Schematic diagram of the response system, showing the feedback subcircuit that couples the response circuit to the drive circuit.

The rate equations that describe the dynamics in both circuits are the same, except for parameter mismatch of about $1 \%$ and the coupling term between the circuits which is present in the response circuit equation:

$$
\begin{aligned}
\dot{V}_{1 r}= & -\alpha V_{1 r}-\beta I\left(V_{2 r}\right)-\theta V_{E} \\
& +\stackrel{\varepsilon}{ }
\end{aligned}
$$




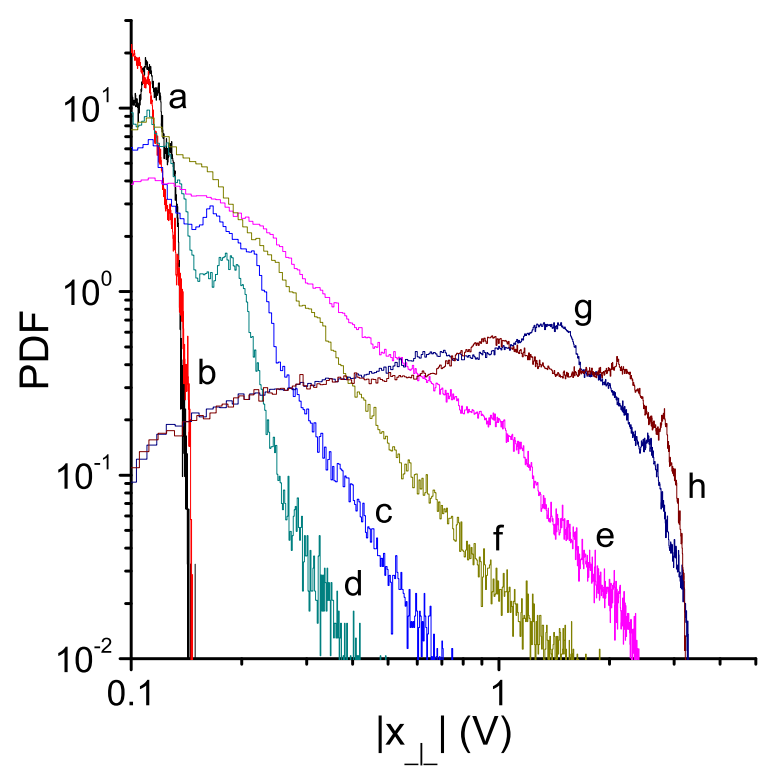

Figure 6: Log-log distribution of the voltages obtained from the error signal $\left|x_{\perp}\right|=\left|V_{1 d}-V_{1 r}\right|+$ $\left|V_{2 d}-V_{2 r}\right|$ time series Figure 5 for different coupling levels: (a) and (b) $\epsilon=1.0$, (c) and (d) $\epsilon=$ 0.6, (e) and (f) $\epsilon=0.4,(\mathrm{~g})$ and $(\mathrm{h}) \epsilon=0.0$. (a), (c), (e) and (g) with external pumping of $A=4.0$ $\mathrm{V}$ and $f=770 \mathrm{~Hz}$ and (b), (d), (f) and (h) with external pumping of $A=4.0 \mathrm{~V}$ and $f=980 \mathrm{~Hz}$.

highly synchronized states. When the coupling is weak $(\epsilon<0.3)$ for both states the oscillations of circuits are practically independent, specially for $\epsilon=0.0$ (Fig. 6(g) and 6(h)) when they are completely independent. Between the situations with strong and weak coupling, i.e., for moderate coupling, the system exhibits attractor bubbling, but it occurs for different levels of coupling depending on which attractor "shape" (again attractor A or $\mathrm{B})$ is used. For $\epsilon=0.6$, we observe in Fig. 6(c) a heavy-tailed structure whereas in Fig. 6(d) we do not observe a heavy-tailed structure, the system is still practically synchronized. For $\epsilon=0.4$ (see Fig. 6(e)) the system has a large error signal, whose bursts of desynchonization are so frequent that we do not consider them as bubbling phenomena, but a signature that the circuits are independent. In Fig. 6(f) $(\epsilon=0.4)$ the system exhibits bubbling, having a heavy-tailed structure. Thus, synchronization is harder for $f=770 \mathrm{~Hz}$ (attractor A) than it is for $f=980 \mathrm{~Hz}$ (attractor $\mathrm{B}$ ). As one can observe attractor $\mathrm{B}$ exhibits bubbling for coupling levels smaller than attractor A. Notice that curves (e) and (c) of Fig. 6 are higher than their respectives (f) and (d), with the same values of $\epsilon$.

The occurrence of attractor bubbling is due to a region of instability in the phase space of the chaotic system. Thereby, when a trajectory of the system gets close to this unstable region there is a certain probability that the trajectory of the drive circuit will be repelled in a direction while the response system will be repelled in a different one, generating the bursts of desynchronization shown in Fig. 6. Hence, when our coupled chaotic oscillators approach the region with $V_{1}=V_{2}=0$ $\mathrm{V}$ there is a nonzero probability that noise or parameter mismatch will throw the drive oscillator to one sign of $V_{2}$ and at same time the receiver oscillator to the other sign of $V_{2}$, thus causing a desynchronization event (attractor bubble). As the trajectories in phase space of attractor A pass more often in the region around $V_{1}=V_{2}=0 \mathrm{~V}$ than the trajectories of attractor $\mathrm{B}$, the probability of attractor A exhibiting a burst is greater than that of attractor B. Thus attractor bubbling occurs for attractor B at coupling strengths smaller than those necessary for attractor A.

\section{Conclusions}

Studying the coupling between two chaotic electronic circuits we showed the existence of attractor bubbling for two different chaotic attractors and for moderate coupling strengths. Analysing the empirical distribuitions of error signal of the system we find that attractor bubbling generates heavy-tailed distributions that are reasonably approximated by truncated power-laws. The attractor shape interferes with the behavior of the error signal of two coupled chaotic systems. As one of the attractors passes more often close to the unstable region of phase space compared to the other one, it requires stronger coupling to exhibit bubbling.

\section{Acknowledgements}

We thank Brazilian Agencies Conselho Nacional de Desenvolvimento Científico e Tecnológico $(\mathrm{CNPq})$ and Coordenação de Aperfeiçoamento de Pessoal de Nível Superior (CAPES) for financial support.

\section{References}

Acebrón, J. A., Bonilla, L. L., Vicente, C. J. P., Ritort, F. and Spigler, R. (2005). The kuramoto model: A simpler paradigm for synchronization phenomema, Rev. Mod. Phys. 77: 137-185. DOI: 10.1103/RevModPhys.77.137

Ashwin, P., Buescu, J. and Stewart, I. (1994). Bubbling of attractors and synchronisation of chaotic oscillators, Phys. Lett. A 193(2): $\quad 126-139 . \quad$ DOI: 10.1016/0375-9601(94)90947-4

Boccaletti, S., Kurths, J., Osipov, G., Valladares, D. and Zhou, C. (2002). The synchronization of chaotic systems, Physics Reports 366(1): 1-101. DOI: $10.1016 /$ S0370-1573(02)00137-0 
Cavalcante, H. L. D. S., Oriá, M., Sornette, D., Ott, E. and Gauthier, D. J. (2013). Predictability and suppression of extreme events in a chaotic system, to be published .

Gauthier, D. J. and Bienfang, J. C. (1996). Intermittent loss of synchronization in coupled chaotic oscillators: Toward a new criterion for high-quality synchronization, Phys. Rev. Lett. 77(9): 1751-1754. DOI: 10.1103/PhysRevLett.77.1751

Gonçalves, C. and Neto, L. (2011). Electrical implementation of a complete synchronization dynamic system, Journal of Physics: Conference Series, Vol. 285, IOP, IOP Publishing, p. 012013.

Heagy, J. F., Carroll, T. L. and Pecora, L. M. (1995). Desynchronization by periodic orbits, Phys. Rev. E 52(2): R1253-R1256. DOI: 10.1103/PhysRevE.52.R1253

Junior, G. F. O., Cavalcante, H. L. D. S., di Lorenzo, O., Chevrollier, M., de Silans, T. P. and Oriá, M. (2013). Tunable power law in the desynchronization events of coupled chaotic electronic circuits, to be published .

Kocarev, L. and Parlitz, U. (1996). Generalized synchronization, predictability, and equivalence of unidirectionally coupled dynamical systems, Phys. Rev. Lett. 76: 1816-1819. DOI: 10.1103/PhysRevLett.76.1816

Pecora, L. M. and Carroll, T. L. (1990). Synchronization in chaotic systems, Phys. Rev. Lett. 64(8): 821-824. DOI:

10.1103/PhysRevLett.64.821

Rosenblum, M. G., Pikovsky, A. S. and Kurths, J. (1996). Phase synchronization of chaotic oscillators, Phys. Rev. Lett. 76: 1804-1807. DOI: 10.1103/PhysRevLett.76.1804

Rulkov, N. F., Sushchik, M. M. and Tsimring, L. S. (1995). Generalized synchronization of chaos in directionally coupled chaotic systems, Phys. Rev. E 51: 980-994. DOI: 10.1103/PhysRevE.51.980

Strogatz, S. (1994). Nonlinear Dynamics and Chaos, Perseus Books, Cambridge, Massachusetts.

Venkataramani, S. C., Hunt, B. R., Ott, E., Gauthier, D. J. and Bienfang, J. C. (1996). Transitions to bubbling of chaotic systems, Phys. Rev. Lett. 77(27): 5361-5364. DOI:

10.1103/PhysRevLett.77.5361 